\title{
Herbal medicines on the spot: back to the green essentials
}

\begin{abstract}
Plants have always been an attractive source of new molecular entities, many of which hold potential for therapeutic purposes. From an analytical point of view, they pose a challenge since their compounds act in a synergistic way. Hence, powerful analytical techniques and data processing tools are put in place to unravel the green mystery. The aim of this mini review is to revisit key topics in the field of herbal medicines, such as main concepts of the trade, strategies to analyze complex mixtures, cutting-edge technologies and data processing tools to serve this purpose.
\end{abstract}

Keywords: herbal medicines, high-throughput analytical technologies, plant metabolomics, dereplication
Volume 8 Issue 3 - 2019

\author{
Silvia E Lucangioli, ${ }^{1,3}$ Marcelo LWagner, ${ }^{2}$ \\ Cecilia B Dobrecky ${ }^{1,2}$ \\ 'Universidad de Buenos Aires, Facultad de Farmacia y \\ Bioquímica, Buenos Aires, Argentina \\ ${ }^{2}$ Universidad de Buenos Aires, Facultad de Farmacia y \\ Bioquímica, Buenos Aires, Argentina \\ ${ }^{3}$ CONICET - Universidad de Buenos Aires, Facultad de \\ Farmacia y Bioquímica, Buenos Aires, Argentina
}

Correspondence: University of Buenos Aires, Faculty of Pharmacy and Biochemistry. Department of Pharmaceutical Technology. Chair of Pharmaceutical Technology I, Department of Pharmacology, Chair of Pharmacobotany, Buenos Aires, Argentina,Tel 54 II 52874462,Email Icdobrec@ffyb.uba.ar

Received: March 22, 2019 | Published: May 02, 2019
Abbreviations: HPLC, high performance liquid chromatography; UHPLC, ultra-high-pressure liquid chromatography MS, mass spectrometry; TOF, time of flight; IT, ion-trap; FT-ICR, fourier transform ion cyclotron resonance; MALDI, matrix-assistedlaser-desorption ionization; IMS, imaging mass spectrometry; NMR, nuclear magnetic resonance; qNMR, quantitative NMR; DOSY, diffusion ordered spectroscopy; SPE, solid-phase extraction; PCA, principal component analysis; HR-MAS, high resolution-magical angle spinning; CE, capillary electrophoresis; CZE, capillary zone electrophoresis; MEKC, micellar electrokinetic chromatography; NACE, nonaqueous capillary electrophoresis; PLS, partial least squares; UNPD, universal natural product database; HCA, hierarchical clustering

\section{Introduction}

Plants have been inextricable intertwined with the history of humanity. They are an inextinguishable source of compounds, most of which serve as templates for their synthetic counterparts. "Traditional medicine can be defined as the sum of the total knowledge, skill and practices based on the theories, beliefs and experiences indigenous to different cultures, whether explicable or not, used in the maintenance of health as well as in the prevention, diagnosis, improvement or treatment of physical and mental illness. Herbal medicines are naturally occurring, plant-derived substances with minimal or no industrial processing that have been used to treat illness within local or regional healing practices". ${ }^{1}$ It is generally accepted that their use has been validated through continuous utilization. The therapeutic effect of plant extracts is mostly based on a synergistic effect of their multiple components instead of the classic single-compound-based approach. ${ }^{2}$ From an analytical standpoint, this poses a double challenge. On one hand, powerful tools are needed for the rapid identification of known components to provide an effective quality control. On the other hand, once the known analytes have been identified, attempts should be made for the discovery of the new ones. In this sense, it is of the utmost importance to get as much as information as possible in every single run.

\section{Some ground rules}

The ongoing innovation in the field of analytical tools has coined a plethora of terms related to the different approaches in which a complex sample (like a plant extract) is to be studied. The amount of data generated as a result of every single chromatographic run has driven a boost in data analysis strategies as well. In this ever-evolving context, some wording clarification is needed.

The advent of the "Omics revolution" marked a paradigmatic change in scientific philosophy and gave way to several approaches in which the term is used as a suffix to serve multiple purposes. In this way, metabolomics can be defined as "the technology geared towards providing an essentially unbiased, comprehensive qualitative and quantitative overview of the metabolites (with a special emphasis on small molecules) present in an organism". ${ }^{3}$ Plant metabolism is especially challenging in terms of understanding biochemical pathways and their regulation and even nowadays, many essential aspects remain unknown. In the words of Oliver Fiehn, plant biochemistry can still be defined as "unidentified compounds derived from undefined pathways and with unknown function". ${ }^{4}$ Despite significant advances, many "green" enigmas are yet to be discovered. In this complex scenario, the initial stage of our search is basically, nontargeted.

Some additional terms, now broadly employed, should be dealt with as well. In this sense, plant metabolic fingerprinting is defined as a high throughput qualitative screening of the metabolic composition in which the primary goal is to perform a sample comparison and 
discrimination analysis. Generally, there is no initial intention of identifying compounds. The whole procedure from sample preparation to detection is meant to be quick and easy, if possible. It is usually a head start to metabolic profiling. The latter refers to the identification and quantification of metabolites, which is commonly applicable to a limited number of selected components. ${ }^{3}$

Once a general metabolomics approach is carried out or based on previous knowledge, it is possible to narrow down the initial search and focus on a selected group of metabolites by means of a dedicated extraction and detection. This is called a targeted analysis. ${ }^{3}$

The first definition of "dereplication" applied to "a process of quickly identifying known chemotypes", ${ }_{5}^{5}$ or, as in this case, known analytes. Since then, the term has evolved, and it is considered as a strategy to circumvent the efforts of isolating known compounds. Moreover, it is based on metabolite profiling and is carried out as the first step in the treasure hunt for novel molecular entities. ${ }^{6}$

\section{The intrinsic polypharmacological nature of herbal medicines}

The traditional approach used in phytochemical research was based on isolating and identifying a single compound, usually by means of a bioguided fractionation strategy. The boost in technological development applied to chromatography and spectroscopic methods, such as mass spectrometry and nuclear magnetic resonance, served this purpose as bioactive compounds continue to play a key role in drug discovery. This gave way to the concept of a single plant metabolite as a "magic bullet" to treat a disease. ${ }^{7}$ The "one drug, one target, one disease" concept has been the prevailing motto in pharmaceutical research and therapeutics. However, the complex etiology and multifactorial nature of certain chronic diseases such as cancer, atherosclerosis and diabetes has shifted the course. This has also been driven by the need to reduce the cost, time and effort involved in isolating a single compound, which often, cannot account for the whole therapeutic effect. ${ }^{8}$ Treatment of these conditions requires modulation of multiple biological targets to reinstate physiological balance and display therapeutic efficiency. Herbal medicines are essentially a combination of multitarget drugs that tackle several biological targets and promote additive or synergistic effects. This is termed polypharmacology. ${ }^{9}$

\section{High-throughput analytical technologies in a glimpse}

The vast diversity of metabolites within the plant kingdom, the need to understand their complex interactions and potential application for therapeutics have driven a phenomenal development peak which in turn led to this era of high-throughput analytical technologies. These made possible to tackle large amounts of compounds in complex mixtures, such as plant extracts in a manageable way. Hyphenation has been a key player in this process, as it combines multiple features of every equipment into a single array.

HPLC (and now UHPLC) remains as the most popular technique for plant extract analysis, and the possibility of combining multiple detection modes makes it the ideal choice. The traditional approach was based on HPLC coupled to a photodiode array detector. However, mass spectrometry (MS) in both configurations, that is, as a standalone array or hyphenated to UHPLC, provides high sensitivity and speed and it gives information on molecular mass, abundance, adduct formation and fragmentation pattern.

A broad classification of mass analyzers is related to resolution. In this sense, a general classification could be made, namely high (HRMS) and low resolution (or nominal mass) instruments and the basis for such distinction lies in the resolving power. In this sense, high resolution mass spectrometers generally provide resolving power greater than 10,000 (such as Time-of-flight analyzers) while ultrahigh-resolving power instruments are above 100,000 (as is the case with Fourier transform instruments such as Orbitrap and ion cyclotron resonance). Ionization is a key step in mass spectrometry. The way in which analyte ionization takes place is based on the physical state of the analyte molecules, that is, a) gas or vapor, b) liquid or in solution, c) solid or dry on a target. Electron ionization and chemical ionization are examples of gas-phase ionization techniques which are frequently used in GC-MS. This approach is particularly useful for volatiles or derivatized compounds. ESI, which is a liquid-phase ionization technique extensively used on LC-MS, is by far the most used ionization mode, both in negative and positive variants and a variety of mass analyzers are available as well, such as time of flight (TOF), quadrupole, ion-trap (IT), Fourier Transform ion cyclotron resonance (FT-ICR) and the recent Orbitrap. ${ }^{10}$

Another aspect of relevance is the possibility of performing a multistage mass spectrometry, which gives additional information on the analyte. This is also called tandem mass spectrometry and it is extensively used for structure elucidation.

A literature survey shows that a broad range of secondary metabolites in plant natural products, including phenolics, flavonoids, terpenoids, alkaloids, coumarin, and saponins, among others, have been analyzed using ESI coupled to different HRMS instruments such as TOF, Q-TOF, IT-TOF, Linear trap quadrupole (LTQ)-Orbitrap, Q-Orbitrap or Fourier-transform ion cyclotron resonance (FT-ICR), both as stand-alone or hyphenated configurations. HRMS instruments based on TOF-MS analyzers are the most extensively reported for the analysis of medicinal plants products. In particular, the hybrid Q-TOF-MS instruments have been largely adopted as powerful tools for untargeted analysis of complex plant extracts, due to their capability of providing accurate mass data, and structural information from HR-MS/MS fragmentation. ${ }^{11}$ An integrated strategy of UHPLC/ LTQ-Orbitrap-MS was used for the characterization and identification of the bioactive molecules from Uncaria rhynchophylla. A total of 92 alkaloids were characterized, 56 of which are potential new alkaloids for this species. A novel approach was carried out by HPLC-HRMS technique and applied to Epimedium metabolites. A combined MS strategy using both phytochemical and biological approaches was used to screen the chemical constituents of Glycyrrhiza uralensis (licorice). A total of 122 compounds were identified by MS from the roots and rhizomes of licorice. ${ }^{12}$

Matrix-assisted-laser-desorption ionization (MALDI) and desorption electrospray ionization (DESI) are examples of solidphase or surface ionization techniques. The first one is a gentle ionization technique, usually applicable to detect and characterize analytes with high molecular weights. It has been applied to determine the molecular weight of some natural products, to identify chemical structures as well as metabolomic studies and recently, it has been employed to establish tissue distribution of metabolites by MALDI imaging. The most common analyzers applied to MALDI are IT, 
quadrupole, Orbitrap, FT-ICR and TOF. ${ }^{13}$ There are some limitations regarding its application to low molecular weight compounds $(<\mathrm{m} / \mathrm{z}$ $1500 \mathrm{Da}$ ) but several strategies have been deployed to circumvent this problem. As a result, MALDI has been successfully used for the analysis of secondary metabolites such as flavonoids, phenolics, lactones, triterpenes, alkaloids, condensed tannins, anthocyanins. ${ }^{14}$ As for metabolomics, a protocol has been reported for plant analysis by MALDI-TOF. ${ }^{15}$ Imaging mass spectrometry (IMS) has been positioned itself in the field of "spatial metabolomics". ${ }^{16}$ MALDI imaging is not routinely applicable to plant analysis since there are many difficulties in preparing the samples. However, it can provide valuable information about metabolite and tissue distribution maps. ${ }^{13}$ DESI offers some advantages in comparison to other IMS techniques as it does not require extensive sample preparation, is rapid and minimally destructive and is performed at ambient conditions. It enables the accurate view of molecules in tissues. It has been applied on the imprints of leaves, flowers and petals, fruits and seeds. As an example, Hypericum perforatum leaves and petals were analyzed to study the spatial distribution of hypericin and hyperforin. ${ }^{16}$

Nuclear magnetic resonance (NMR), although less sensitive than MS, is widely employed for metabolomics and works as a complementary technique. The different strategies applied to herbal medicines could be classified as 1) conventional NMR, 2) hyphenated NMR, 3) quantitative NMR (qNMR), 4) NMR based metabolomics and 5) Diffusion ordered spectroscopy (DOSY). 1D and 2D conventional NMR have found application without fractionation or isolation steps. ${ }^{17}$ A standard ${ }^{1} \mathrm{H}$ NMR experiment provides general information about the relative number of hydrogen atoms, however insufficient for complete structural elucidation. This complements with ${ }^{13} \mathrm{C}$ NMR experiments and 2D NMR for the correct assignment of all ${ }^{1} \mathrm{H}$ and ${ }^{13} \mathrm{C}$ signals. 2D NMR spectra are produced by homonuclear $\left({ }^{1} \mathrm{H}-{ }^{-1} \mathrm{H}\right.$ correlated experiments such as COSY, applied to neighboring protons; and TOCSY, which identifies protons belonging to the same spin system) and heteronuclear experiments (HSQC correlates ${ }^{13} \mathrm{C}$ nuclei with ${ }^{1} \mathrm{H}$ nuclei within a molecule by means of the one-bond coupling between them; and $\mathrm{HMBC}$, which correlates proton nuclei with carbon nuclei that are separated by more than one bond).

This has been successfully applied to the analysis of Hypericum perforatum in which flavonols, phloroglucinols, shikimic and chlorogenic acids could be identified. Also, a resourceful strategy has been applied to specimens from the Lamiaceae family for the identification of caffeic acid and rosmarinic acid without the need of resorting to conventional chromatographic separation. ${ }^{18}$ NMR has also been hyphenated to HPLC which enables separation, isolation and structure elucidation in one step. This has found application in natural product research but has several disadvantages that hamper its use. While NMR is a universal detection method, it requires sufficient analyte concentration, and this is a limitation if one considers the amount of secondary metabolites in the column eluates. Also, deuterated solvents need to be used. A breakthrough in the field was the surge of an on-line solid-phase extraction (SPE) add-on to the HPLC-NMR systems. This eliminated the problem of using HPLC solvents compatible with NMR, increased sensitivity and consequently significantly reduced NMR acquisition time, the analytes are enriched by a multiple trapping process and the cost is significantly lower. Following chromatographic separation and detection by $\mathrm{DAD} / \mathrm{MS}$, the analytes of interest are retained on SPE cartridges, dried and subsequently eluted with a deuterated solvent into an NMR probe. This has been applied to the analysis of Clausena excavata Burm f., Androsace umbellata (Lour.) Merr., Oxalis corniculata L. and Trachelospermum jasminoides (Lindl.) Lem to identify four non-tannin inhibitors of necrosis enzymes. ${ }^{19}$ qNMR is a non-destructive method and is more accurate and precise than conventional chromatographic methods as quantitation is intrinsically inherent to NMR. No expensive chemical standard is needed for calibration curves and it provides both qualitative and quantitative information. Kavalactones have been quantified in kava-kava extracts using maleic acid as standard; anthracene was used for the quantitation of ephedrine analogues in Ephedra and cannabinoids in Cannabis sativa.${ }^{17}$ Regarding metabolomics, NMR fulfills all the requirements as it is a high-throughput, quantitative, robust and highly reproducible analytical technique. However, the complexity of the spectra of plant extracts often requires multivariate data analysis for discriminating diagnostic signals. This approach has been applied in combination with principal component analysis (PCA) to comparison of commercial preparations of St. John's wort. 2D DOSY ${ }^{1} \mathrm{H}-\mathrm{NMR}$ provides information on the sample and a virtual separation of its components in the mixture. One dimension depicts conventional chemical shifts and the other, diffusion coefficients. It has been used for the analysis of complex matrices such as Camellia sinensis teas and for the differentiation of Ginseng roots from various origins. ${ }^{10} 3 \mathrm{D}-\mathrm{NMR}$ experiments are also feasible and the surprising high resolution-magical angle spinning (HR-MAS) which allows for direct sample analysis with minimum manipulation. NMR holds a promising potential that is yet to be fully exploited. ${ }^{20}$

Capillary electrophoresis (CE) has also been applied to plant extract analysis. It can be coupled to a wide range of detectors such as UV or DAD, electrochemical detection and MS, among others. CE has several advantages compared to other analytical methodologies that comprise minute sample requirement, low-consumption of organic solvents, automation and an eco-friendly methodology. Capillary zone electrophoresis (CZE) is the traditional approach in which analyte separation is based on mass-to-charge ratio. It has been extensively used for herbal medicine analysis since it is the standard operation mode. Examples of its application are related to wine analysis of flavonoids, Flos chrysanthemi and indici. Micellar electrokinetic chromatography (MEKC) is a hybrid between electrophoresis and chromatography and makes use of a surfactant as a component of the background electrolyte. This provides an additional separation mechanism, based on analyte partition in the micelle and electromigration. ${ }^{21,22}$ An example of the latter is related to the analysis of Ligaria cuneifolia extracts by a novel dual cyclodextrin MEKCDAD methodology. ${ }^{23}$ Microemulsion electrokinetic chromatography (MEEKC) applies microemulsions as the background electrolyte to improve the solubility of highly lipophilic compounds. Zhao et al., ${ }^{24}$ reported the analysis of alpinetine and cardamonin in Alpinia katsumadai by means of MEEKC combined with microwave-assisted extraction. Hyphenation with MS is highly employed, especially with ESI. A recent report makes use of the nonaqueous capillary electrophoresis (NACE) which is very well suited for online coupling with MS because of the relatively high volatility and low surface tension of most organic solvents. The NACE-ESI-MS/MS was applied for the separation, identification and quantitation of physcion, chrysophanol, and aloe-emodin in rhubarb. ${ }^{25}$

\section{Data analysis: the end of the mystery?}

"Raw data is nothing but a poor relative of information and information is itself a giant leap away from knowledge". With the 
advent of modern analytical techniques, a huge amount of information is generated, which holds the key to unlock the mystery of plant chemistry. The way in which this information is processed and understood, remains a crucial piece in this complex puzzle. In order to do so, new tools must be developed for an effective in silico data handling. This encompasses a set of informatic tools to 1) collect and preprocess the data for the direct comparison of datasets, 2) process and mine data in order to extract those components of interest, 3) a visual strategy to present complex data in a comprehensive way and 4) effective databasing for appropriate data storage. In the arduous path to translate abstract machine output into accessible information and subsequent new knowledge, an effective discriminant analysis should follow data preprocessing. This comprises some statistical filtering before differential comparisons can be made and reliable conclusions drawn. Hence, unsupervised approaches for discriminatory analyses such as PCA and hierarchical clustering (HCA) or supervised approaches such as partial least squares (PLS) or SIMCA are frequently used. Data visualization is a key aspect that needs special consideration. Tools have been developed to that end such as KEGG and MetNet. ${ }^{3}$ There are a few plant-specific databases with curated information on chemical composition and distribution across plant species such as KNApSAcK, the Universal Natural Product Database (UNPD) or BioPhytMol, to name a few. Bioinformatic tools are needed to assist in metabolomic data interpretation, especially for visualization and metabolite mapping into pathways and biological context and for the integration of the other "omic" approaches stored in different platforms so as to take the leap from data to knowledge. ${ }^{26}$

\section{Conclusion}

Our forebears used to have an intuitive understanding of nature. At present, we need to provide scientific grounds to support this traditional use and try to understand the delicate balance of compound synergy which is inherent to herbal medicines. Medicinal plants still hold great promise to provide answers for our more pressing questions both in drug discovery and therapeutics. It is imperative to improve current bioinformatic tools to integrate all the available information related to metabolomics for a holistic approach. There is still a long way to go and many "green" mysteries to unravel.

\section{Acknowledgments}

None.

\section{Conflicts of interest}

The authors declare no conflicts of interest.

\section{References}

1. Tilburt J, Kaptchuk T. Herbal medicine research and global health: an ethical analysis. Bull World Health Organ. 2008;86(8):594-599.

2. Thomford N, Dimakatso A, Rowe A, et al. Natural Products for Drug Discovery in the $21^{\text {st }}$ Century: Innovations for Novel Drug Discovery. Int J Mol Sci. 2018;19(6):E1578.

3. Hall R. Plant metabolomics: from holistic hope, to hype, to hot topic. New Phytol. 2006;169(3):453-468.

4. Oliver Fiehn. 1st Metabolomics Conference. University of California: Japan; 2005.
5. Hubert J, Nuzillard J, Renault J. Dereplication strategies in natural products research: How many tools and methodologies behind the same concept? Phytochem Rev. 2017;16(1):55-95.

6. Borges R, Taujale R, Santana de Souza J, et al. Dereplication of plant phenolics using a mass spectrometry database independent method. Phytochem Anal. 2018;29(6):601-612.

7. Li F, Weng J. Demystifying traditional herbal medicine with modern approaches. Nat Plants. 2017;3:17109.

8. Zhou X, Seto S, Chang D, et al. Synergistic Effects of Chinese Herbal Medicine: A Comprehensive Review of Methodology and Current Research. Front Pharmacol. 2016;7:201.

9. Proschak E, Stark H, Merk D. Polypharmacology by Design: A Medicinal Chemist's Perspective on Multitargeting Compounds. J Med Chem. 2019;62:420-444.

10. Niessen W, Falck D. Introduction to Mass Spectrometry, a Tutorial. Germany: Wiley-VCH Verlag GmbH \& Co; 2015:1-54.

11. Álvarez-Rivera G, Ballesteros-Vivas D, Parada-Alfonso F, et al. Recent applications of high resolution mass spectrometry for the characterization of plant natural products. TrAC Trends in Analytical Chemistry. 2019;112:87-101.

12. Zhang A, Sun H, Yan G, et al. Recent developments and emerging trends of mass spectrometry for herbal ingredients analysis. TrAC Trends in Analytical Chemistry. 2017;94:70-76.

13. Silva R, Lopes N, Silva D. Application of MALDI Mass Spectrometry in Natural Products Analysis. Planta Med. 2016;82(8):671-689.

14. Mandal A, Singha M, Addy P, et al. Laser desorption ionization mass spectrometry: Recent progress in matrix-free and label-assisted techniques. Mass Spectrom Rev. 2019;38(1):3-21.

15. Ernst M, Silva D, Silva R, et al. A metabolomic protocol for plant systematics by matrix-assisted laser-desorption/ionization time-of flight mass spectrometry. Analytica Chimica Acta. 2015;859:46-58.

16. Parrot D, Papazian S, Foil D, et al. Imaging the Unimaginable: Desorption Electrospray Ionization - Imaging Mass Spectrometry (DESI-IMS) in Natural Product Research. Planta Med. 2018;84(9-10):584-593.

17. Gilard V, Balayssac S, Malet-Martino M, et al. Quality control of herbal medicines assessed by NMR. Curr Pharm Anal. 2010;6:234-245.

18. Andersen O, Markham R. Spectroscopic techniques applied to flavonoids in Flavonoids: chemistry, biochemistry and applications. USA: Taylor \& Francis; 2005 .

19. Cies L, Moaddel R. Comparison of analytical techniques for the identification of bioactive compounds from natural products. Nat Prod Rep. 2016;33(10):1131-1145.

20. Mahrous E, Farag M. Two dimensional NMR spectroscopic approaches for exploring plant metabolome: a review. $J A d v$ Res. 2015;6(1):3-15.

21. Wu T, Yu C, Li R, et al. Minireview: Recent advances in the determination of flavonoids by capillary electrophoresis. Instrum Sci Technol. 2018; 364-386.

22. Ma H, Bai Y, Li J, et al. Screening bioactive compounds from natural product and its preparations using capillary electrophoresis. Electrophoresis. 2018;39(1):260-274.

23. Dobrecky C, Flor S, López P, et al. Development of a novel dual CD MEKC system for the systematic flavonoid fingerprinting of Ligaria cuneifolia (R. et P.) Tiegh. -Loranthaceae -extracts. Electrophoresis. 2017;38(9-10):1292-1300. 
24. Zhao Y, Lü H, Xu L. Rapid Analysis of Alpinetin and Cardamonin in Alpinia katsumadai Hayata Using Short-End Injection Microemulsion Electrokinetic Capillary Chromatography Combined with MicrowaveAssisted Extraction. Chromatographia. 2017;80(2):347-352.

25. Cheng J, Wang L, Liu W, et al. Quantitative Nonaqueous Capillary Electrophoresis-Mass Spectrometry Method for Determining Active Ingredients in Plant Extracts. Anal Chem. 2017;89(3):1411-1425.
26. Pérez de Sousa L, Naake $T$, Tohge $T$, et al. From chromatogram to analyte to metabolite. How to pick horses for courses from the massive web resources for mass spectral plant metabolomics. Gigascience. $2017 ; 6(7): 1-20$. 